\title{
Syndecans promote mycobacterial internalization by lung epithelial cells
}

\author{
Natalie Zimmermann, ${ }^{1,2, \dagger}$ Hiroyuki Saiga ${ }^{1, \neq, \dagger}$ \\ Erica Houthuys ${ }^{1, \S, \dagger}$ Pedro Moura-Alves, ${ }^{1}$ Anne Koehler, ${ }^{1}$ \\ Silke Bandermann, ${ }^{1}$ Anca Dorhoi ${ }^{1}$ and \\ Stefan H.E. Kaufmann ${ }^{1 *}$ \\ ${ }^{1}$ Department of Immunology, Max Planck Institute for \\ Infection Biology, Berlin, Germany. \\ ${ }^{2}$ Research Group of Molecular Immunology, Max Planck \\ Institute for Infection Biology, Berlin, Germany.
}

\section{Summary}

Pulmonary tuberculosis (TB) is an airborne disease caused by the intracellular bacterial pathogen Mycobacterium tuberculosis (Mtb). Alveolar epithelial cells and macrophages are the first point of contact for $M t b$ in the respiratory tract. However, the mechanisms of mycobacterial attachment to, and internalization by, nonprofessional phagocytes, such as epithelial cells, remain incompletely understood. We identified syndecan 4 (Sdc4) as mycobacterial attachment receptor on alveolar epithelial cells. Sdc4 mRNA expression was increased in human and mouse alveolar epithelial cells after mycobacterial infection. Sdc4 knockdown in alveolar epithelial cells or blocking with anti-Sdc4 antibody reduced mycobacterial attachment and internalization. At the molecular level, interactions between epithelial cells and mycobacteria involved host Sdc and the mycobacterial heparin-binding hemagglutinin adhesin. In vivo, Sdc1/Sdc4 doubleknockout mice were more resistant to Mtb colonization of the lung. Our work reveals a role for distinct Sdcs in promoting mycobacterial entry into alveolar epithelial cells with impact on outcome of TB disease.

Received 15 December, 2015; revised 11 May, 2016; accepted 5 June, 2016. *For correspondence. E-mail kaufmann@ mpiib-berlin.mpg.de; Tel. +49 3028460 500; Fax +49 3028460501.

${ }^{\dagger}$ These authors contributed equally to this work.

${ }^{\ddagger}$ Faculty of Medicine, Kagawa University, Kagawa, Japan

$\S_{\text {iTeos Therapeutics, Gosselies, Belgium }}$

\section{Introduction}

Tuberculosis (TB) is a global health threat with 9.6 new cases and 1.5 million deaths in 2014 (WHO, 2015). This threat is exacerbated by coinfection with the human immunodeficiency virus (HIV) and the emergence of multidrug-resistant strains (WHO, 2015). Mycobacterium tuberculosis (Mtb), the causative agent of TB, is inhaled in small droplets into the alveolar space, where it is internalized through phagocytosis mainly by alveolar macrophages (Guirado et al., 2013; Pieters, 2008). However, mycobacteria also invade non-phagocytic cells such as epithelial cells (Bermudez and Goodman, 1996; Bermudez and Sangari, 2001; Teitelbaum et al., 1999). Mononuclear phagocytes are both habitat of and effectors against mycobacteria whereas lung epithelial cells primarily provide a protective niche (Nishimura et al., 2008; Nouailles et al., 2014; Rivas-Santiago et al., 2008; Saiga et al., 2008). Although epithelial cells are increasingly appreciated as critical players in TB, the molecular crosstalk with mycobacteria promoting attachment to and internalization by epithelial cells remains largely unknown.

Syndecans (Sdcs) are type I transmembrane cell surface proteoglycans, which mediate cell binding, cell signalling and cytoskeletal organization (Bishop et al., 2007; Couchman, 2010). Their extracellular domains are covalently attached to heparan sulfate chains, which bind to a broad spectrum of extracellular molecules (Bartlett and Park, 2010). The Sdc protein family comprises four members (Sdc1-Sdc4): Sdc1 is expressed on epithelial cells and plasma cells, Sdc2 on endothelial cells, Sdc3 in the nervous system and Sdc4 is ubiquitously expressed (Woods et al., 1998). Because Sdcs bind to and immobilize surface and matrix proteins, cytokines and chemokines, they are considered key regulators of numerous biological processes relevant to inflammation. Consequently, their dysregulation is involved in many inflammatory disorders (Gotte, 2003). Sdcs have also been shown to play a central role during infection of host cells by various viral and bacterial pathogens (Bacsa et al., 2011; Bobardt et al., 2003; de Witte et al., 2007; Freissler et al., 2000; Lefevre et al., 2014; Shi et al., 2013). For example, Sdcs are misused as attachment receptors by HIV-1 (Bobardt et al., 2003; de Witte et al., 2007) or by the herpes simplex virus, which up-regulates 
Sdc1 expression thereby facilitating its entry into host cells (Bacsa et al., 2011).

Mycobacterium bovis is the causative agent of cattle TB. Attenuated between 1906 and 1921 by Calmette and Guérin, it has since then been used as a vaccine against TB. $M$. bovis bacille Calmette-Guérin (BCG) is currently the only licensed vaccine against TB (Andersen and Kaufmann, 2014). Although BCG has marginal effect against pulmonary $\mathrm{TB}$, it protects against extrapulmonary disease forms in infants (WHO, 2015). The vaccine BCG is also widely used in experimental studies.

Heparin-binding hemagglutinin (HBHA) is a 199-residue adhesin that is expressed on the surface of both BCG and Mtb (Menozzi et al., 1996). HBHA contributes to mycobacterial attachment to epithelial cells, likely favouring the dissemination of Mtb from the lung to other organs such as spleen and liver (Delogu and Brennan, 1999; Menozzi et al., 1996; Menozzi et al., 1998; Menozzi et al., 2006; Parra et al., 2004; Pethe et al., 2000; Pethe et al., 2001).

In the present study, we identified Sdc4 as an important interaction partner of $M t b$ and BCG with epithelial cells. The knockdown (KD) of Sdc4 in human alveolar epithelial cells reduced mycobacterial attachment and internalization. Moreover, Sdc4 was found to interact with HBHA. Mice lacking both Sdc1 and Sdc4 exhibited reduced colonization of the lung by $M t b$ at early time points post-infection (p.i.). These findings not only elucidate novel molecular entities involved in the crosstalk between mycobacteria and alveolar epithelial cells, but also provide the basis for novel intervention strategies against TB.

\section{Results}

Sdc4 expression is increased in alveolar epithelial cells in response to mycobacterial infection

Upon viral infection, Sdc expression is known to be increased on the surface of host cells (Shafti-Keramat et al., 2003). To assess the role of Sdc in mycobacterial infection, we first investigated the expression of Sdc mRNA in alveolar epithelial cells and macrophages in response to mycobacterial infection. Human alveolar epithelial cell line A549 cells, mouse type-II alveolar epithelial cell line T7 cells and macrophages derived from human monocytic cell line THP-1 were infected with BCG for $24 \mathrm{~h}$, and analysed for Sdc mRNA expression by quantitative real-time PCR. The expression of Sdc4, but not of other Sdcs, was increased in BCG-infected alveolar epithelial cells correlating with the amount of bacteria (Fig. 1; Fig. S1 A). Additionally, in primary mouse bone marrow-derived macrophages (BMDMs), Sdc4 expression was up-regulated upon infection with Mtb (Fig. S2). Thus, Sdc4 mRNA expression is up-regulated in alveolar epithelial cells and BMDM during mycobacterial infection.

Sdc1 and Sdc4 are expressed in the lung of Mtb-infected mice and patients with active TB

The gene expression portal BioGPS database (http:// biogps.org) predicts high expression of both Sdc1 and Sdc4 on human lung cells. To assess Sdc1 and Sdc4 protein distribution during $M$ tb infection, their expression was analysed by immunohistochemistry in lungs from mice aerosol-infected with $M$ tb $\mathrm{H} 37 \mathrm{Rv}$ and compared to uninfected controls. Upon infection, Sdc4 was strongly upregulated on bronchial and alveolar epithelial cells and
Fig. 1. Sdc4 mRNA induction in epithelial cell lines by BCG. A549 cells, T7 cells and THP-1

macrophages were infected with BCG (MOI of 1, 10, 50 and 100) for $24 \mathrm{~h}$, and quantitative real-time PCR was performed. (A) Sdc1 or (B) Sdc4 mRNA expression is shown as relative mRNA level normalized to the corresponding GAPDH level. Data are representative of three independent experiments. ${ }^{*}, P<0.05$; n.s., not significant using the unpaired t-test. Abbreviations: BCG, bacille Calmette-Guérin; Sdc, syndecan.
A
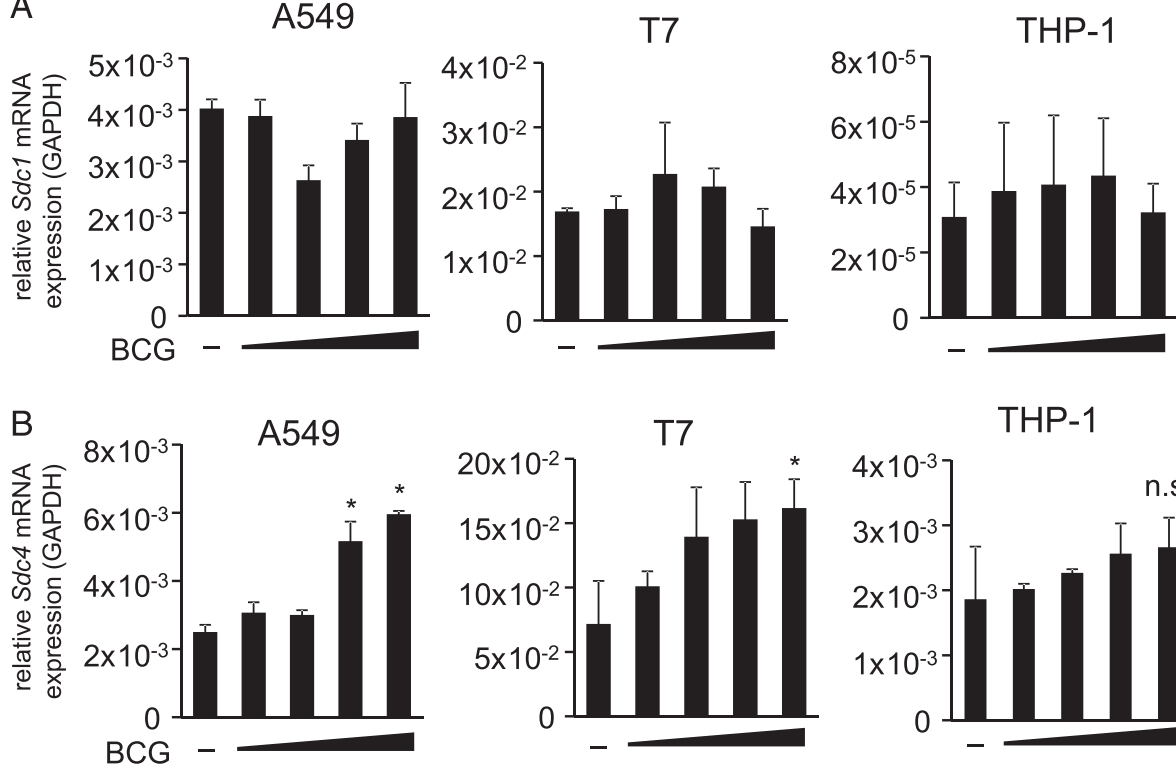

THP-1

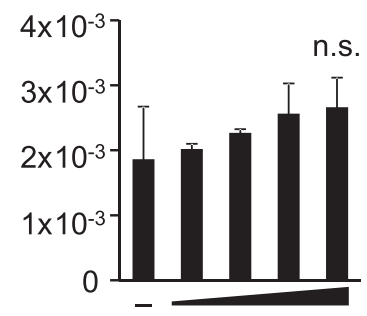


A
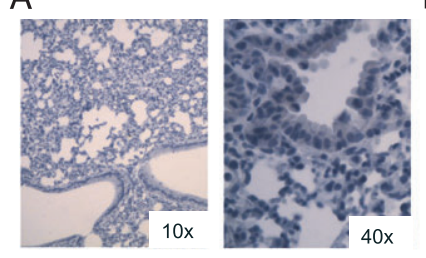

B

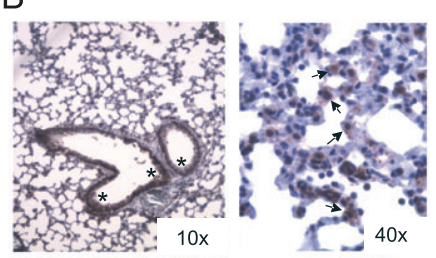

C

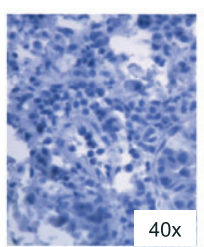

D

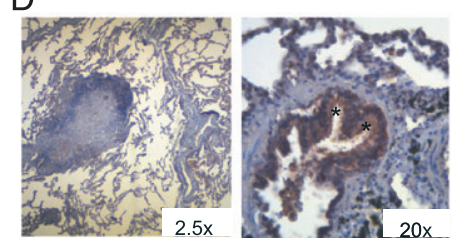

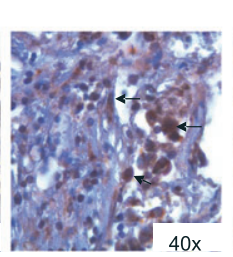

E

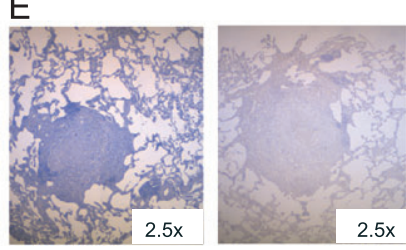

Fig. 2. Sdc4 expression in mouse and human lung tissue.

$A$ and $B$. Mice were infected with low-dose $M t b$ H37Rv. Naive lung $(\mathbf{A})$ and day 15 p.i. are shown (B). Sdc4 staining of bronchial cells (asterisks) and alveolar epithelial cells and macrophages (arrows) is indicated. Lung specimens of a total of three mice were analysed for each condition. C. Rat IgG isotype control on mouse sections. D. Lung biopsies from patients with active TB. Overview (left panel). Sdc4 staining of bronchiolar (middle panel, asterisks) and alveolar epithelial cells and macrophages (right panel, arrows). A total of four specimens were analysed.

E. Rabbit lgG isotype control on human sections as control for Sdc4 staining (left panel) and mouse lgG isotype control on mouse sections as control for Sdc1 staining (right panel). alveolar macrophages compared to naive lungs (Fig. $2 \mathrm{~A}$ and B). Next, Sdc4 was analysed in lung biopsies from patients with active TB. Here, Sdc4 was strongly expressed on epithelial cells in bronchi(oles) and alveoli, as well as on alveolar macrophages (Fig. 2 D). Sdc1 expression was detected in the lungs of both naive and $M t b$-infected mice (Fig. S3 A) and in lung biopsies from patients with active TB (Fig. S3 B). Accordingly, both Sdc1 and Sdc4 proteins were expressed in the lung of $M t b$-infected mice and TB patients.

\section{Sdc4 is involved in mycobacterial attachment to and internalization by lung epithelial cells}

We next constructed Sdc1 and Sdc4 KD cell lines to analyse their role in mycobacterial attachment to epithelial cells (Fig. S1 B). Control, Sdc1 and Sdc4 KD A549 cells were infected with Ds-Red BCG for $3 \mathrm{~h}$, and BCG binding to epithelial cells was determined by confocal microscopy. In comparison to control and Sdc1 KD A549 cells, BCG infection levels of Sdc4 KD cells were markedly reduced (Fig. 3 A, left and middle). Accordingly, colony-forming units (CFUs) recovered from epithelial cell lysates were lower in Sdc4 KD cells than in control and Sdc1 KD cells (Fig. 3 A, right). Both the level of attachment and internalization of BCG bacteria were significantly affected in the Sdc4 KD cell line as revealed by detection of extracellular bacteria via an anti-BCG antibody (Fig. S4). Comparable results were obtained in infection experiments with $M t b$ and A549 control, Sdc1 and Sdc4 KD cells (Fig. S5 A and B). In contrast to epithelial cells, phagocytosis of Mtb H37Rv by Sdc $1^{-/-}$and Sdc4 ${ }^{-/-}$BMDMs was not altered compared to wildtype (WT) macrophages (Fig. S5 C).

Next, we harnessed blocking antibodies specific for Sdc1 and Sdc4 to examine whether blocking of the Sdc receptors inhibited mycobacterial attachment to and internalization by A549 epithelial cells. Upon addition of anti-Sdc4 antibody, the bacterial load in epithelial cells was decreased in a dose-dependent manner whereas the bacterial load of non-treated and anti-Sdc1 antibody-treated cells did not differ (Fig. $3 \mathrm{~B}$ and C). We conclude that Sdc4 is critically involved in mycobacterial attachment to and internalization by alveolar epithelial cells in vitro.

\section{Sdcs bind to heparin-binding hemagglutinin}

$\mathrm{HBHA}$, a mycobacterial surface protein, participates in mycobacterial attachment to epithelial cells (Menozzi et al., 1996). As expected, the internalization rate of epithelial cells was markedly reduced when cells were cultured with an HBHA deletion mutant of BCG (Fig. 4 A). HBHA has been previously shown to interact with heparan sulfate proteoglycans (Dupres et al., 2009; Menozzi et al., 1998; Pethe et al., 2000). Because extracellular domains of Sdcs are composed of heparan sulfate chains (Bartlett and Park, 2010), we determined whether Sdcs bind HBHA. Fluorescently labelled recombinant $\mathrm{HBHA}$ ( $\mathrm{rHBHA})$ was analysed for binding to control, Sdc1 and Sdc4 KD A549 cells. Binding of fluorescently labelled $\mathrm{HBHA}$ to epithelial cells was significantly decreased in the absence of Sdc4 on the cell surface (Fig. 4 B). In contrast, KD of Sdc1 had no effect on binding of $\mathrm{HBHA}$ to epithelial cells. Binding between HBHA and Sdc could be confirmed by ELISA with recombinant proteins (Fig. $4 \mathrm{C}$ ). The affinities of the interactions were in the micromolar range as determined by surface plasmon resonance measurements $\left(\mathrm{K}_{\mathrm{D}}=1.4 \times 10^{-5}, \mathrm{Sdc} 1 ; \mathrm{K}_{\mathrm{D}}=1.3 \times 10^{-5}\right.$, Sdc4) suggesting low-affinity interactions between $\mathrm{HBHA}$ and both Sdc cognates. Although the deletion of either Sdc4 or HBHA decreased the bacterial load in A549 cells, the differential internalization rate between the HBHA mutant and WT BCG was not altered in absence of Sdc4 (Fig. 4 D). Therefore, we consider additional mycobacterial interaction partners for Sdcs beside HBHA.

\section{Enhanced resistance of Sdc1/4 ${ }^{-1-}$ double KO mice to early} Mtb infection

To determine the role of Sdcs in TB, WT, Sdc1 $1^{-/-}$and $\mathrm{Sdc} 4^{-1-}$ single $\mathrm{KO}$ mice as well as $\mathrm{Sdc} 1 / 4^{-1-}$ double $\mathrm{KO}$ 
Fig. 3. Involvement of Sdc4 in BCG attachment to and internalization by epithelial cells.

A. Scramble control and Sdc KD A549 cells were cultured with Ds-Red BCG [MOI of $20($ red)] for $3 \mathrm{~h}$. Cells were stained with phalloidin (green) and nucleus with DRAQ5 ${ }^{\mathrm{TM}}$ (blue). Scale bar represents $50 \mu \mathrm{m}$. Fluorescence intensities of BCG were determined in an average of six random fields using ImageJ software (left and middle). A549 cells were cultured with BCG (MOI of 20 ) for $3 \mathrm{~h}$. Cells were lysed and diluted samples were plated onto agar plates. CFUs were quantified (right).

$B$ and C. A549 cells were treated with $5 \mu \mathrm{g} / \mathrm{ml}$ anti-Sdc1 antibody or $5 \mu \mathrm{g} / \mathrm{ml}$ anti-Sdc-4 antibody for $2 \mathrm{~h}$, and then cultured with Ds-Red BCG [MOI of 20 (red)] for $3 \mathrm{~h}$. Cells were stained with phalloidin (green) and nucleus with $\mathrm{DRAQ5}^{\mathrm{TM}}$ (blue). Scale bar represents $50 \mu \mathrm{m}$. Fluorescence intensity of BCG was determined in an average of six random fields using Image J software (left and middle). A549 cells were treated with anti-Sdc-1 antibody or anti-Sdc-4 antibody $(200 \mathrm{ng} / \mathrm{ml}, 1 \mu \mathrm{g} /$ $\mathrm{ml}, 5 \mu \mathrm{g} / \mathrm{ml}$ ) for $2 \mathrm{~h}$, and then cultured with $\mathrm{BCG}$ (MOI of 20 ) for $3 \mathrm{~h}$. Cells were lysed and diluted samples were plated onto agar plates. Total bacterial counts (CFUs) were quantified (right). Data are representative of three independent experiments. ${ }^{*}, P<0.05$; **, $P<0.01 ;{ }^{* \star \star}, P<0.005$; n.s., not significant using the Bonferroni's posttest (A) or one-way ANOVA (B and C). Abbreviations: $\mathrm{Ab}$, antibody; BCG, bacille Calmette-Guérin; CFU, colonyforming unit; KD, knockdown; Sdc, syndecan.
A

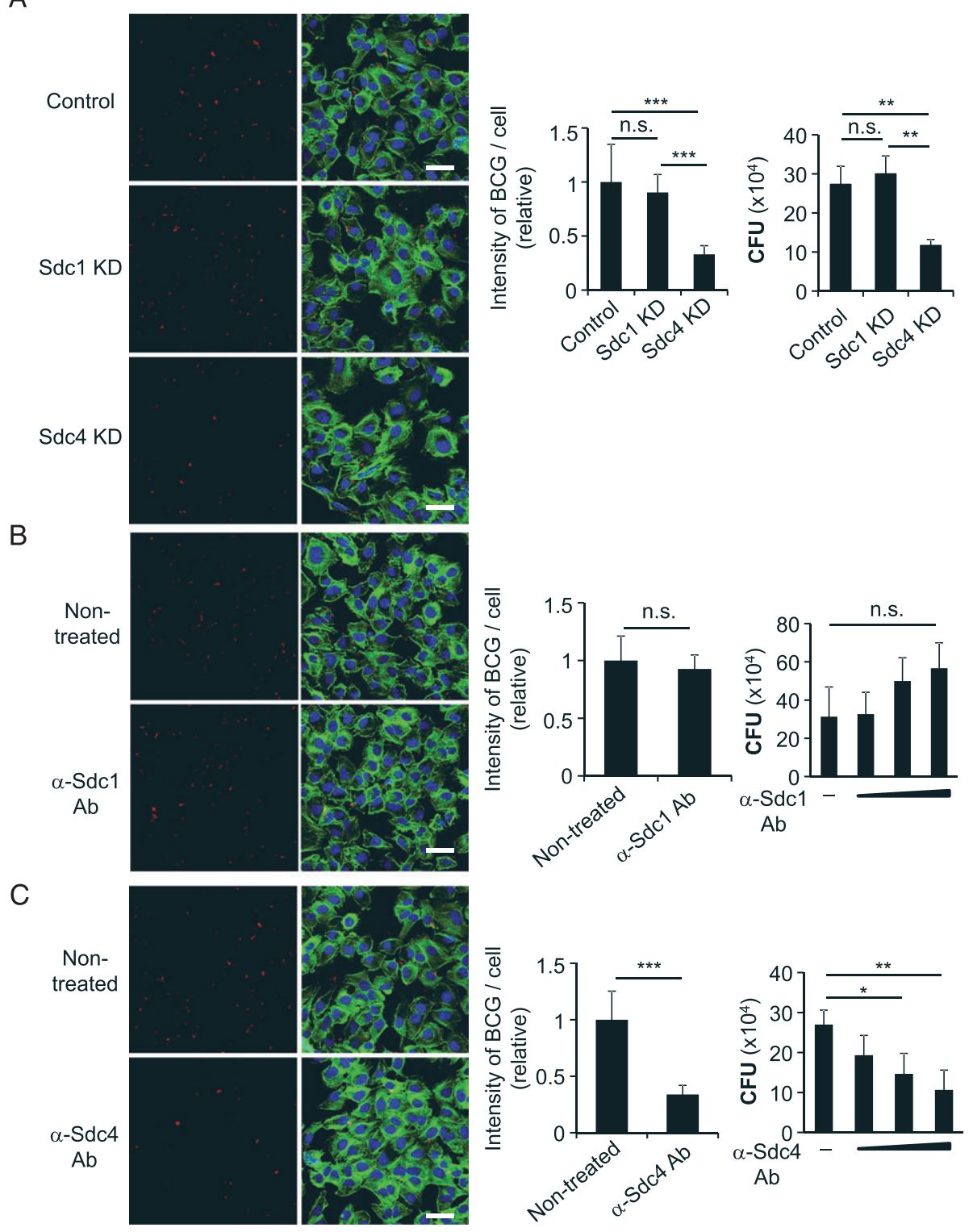

(DKO) mice were infected with a low aerogenic dose of $M t b$ H37Rv and bacterial burdens in lungs were monitored between days 1 and 15 p.i. Overall, CFUs in WT and single KO mice did not differ (Fig. S6). In contrast, CFUs in lungs of Sdc $1 / 4^{-1-}$ DKO animals were significantly reduced over controls at all time points (Fig. $5 \mathrm{~A}$ ). Bacterial dissemination of the bacteria from lung to spleen and liver at days 15 and 21 p.i., however, was not affected (Fig. $5 \mathrm{~B}$ and data not shown). We assume that the early decrease in pulmonary bacterial burden in Sdc1/4 ${ }^{-1-}$ DKO mice was because of reduced internalization by Sdc1/4-expressing cells lining the respiratory tract. To verify this hypothesis experimentally, WT and Sdc1/4 $4^{-1-}$ DKO mice were infected systemically with a high dose of Mtb. In contrast to aerosol infection, bacterial burdens of the lungs of WT and Sdc1/4 ${ }^{-1-}$ DKO mice at day 1 p.i. by the systemic route did not differ (Fig. 5 C). Moreover, the cellular composition of innate immune cells was similar in both naive and Mtb-infected WT and Sdc1/4 ${ }^{-1-}$ DKO mice (Fig. S7). Together, these data demonstrate that Sdc1 and Sdc4 serve as initial contact points for early establishment of pulmonary infection by Mtb.

\section{Immune responses of alveolar epithelial cells to mycobacteria}

To further characterize the role of alveolar epithelial cells in mycobacterial infection, we investigated cell death and cytokine/chemokine secretion of epithelial cells harbouring 
A
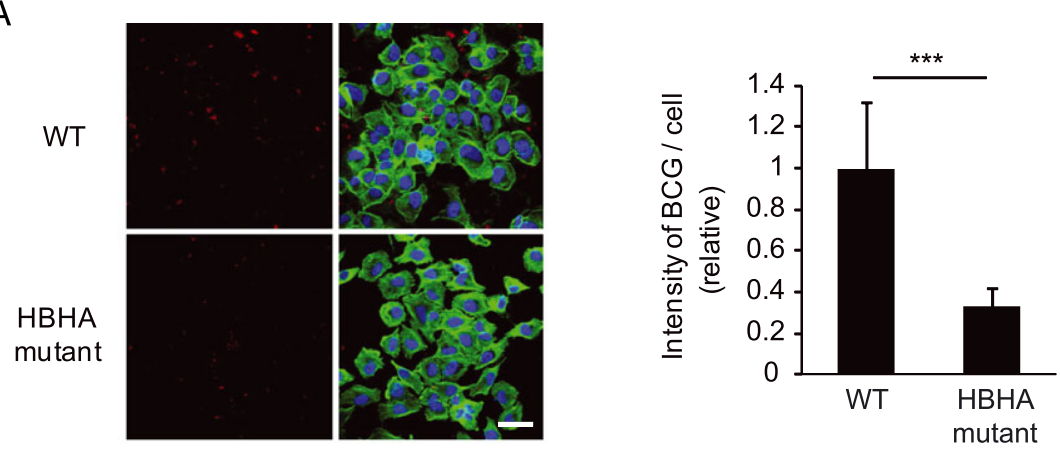

B
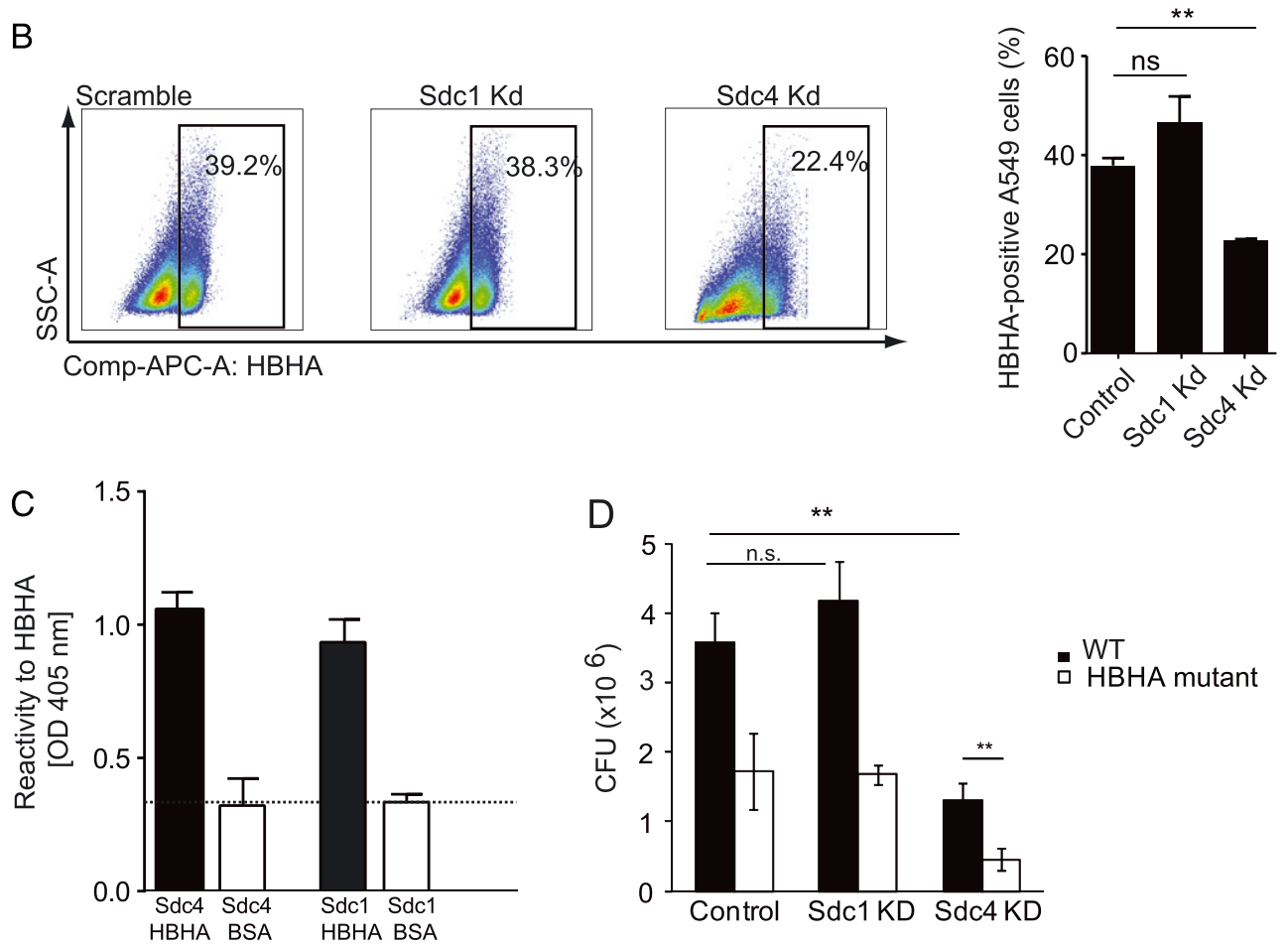

Fig. 4. Involvement of HBHA in mycobacterial attachment to epithelial cells.

A. A549 cells were cultured with Dil-C18-stained WT or HBHA mutant BCG [MOI of 20 (red)] for $3 \mathrm{~h}$. Cells were stained with phalloidin (green) and nucleus with $\mathrm{DRAQ5}^{\mathrm{TM}}$ (blue). Scale bar represents $50 \mu \mathrm{m}$. Fluorescence intensity of BCG was determined in an average of six random fields using Image J software. B. Scramble control and Sdc KD A549 cells were incubated with $2.5 \mu \mathrm{g} / \mathrm{ml}$ of recombinant Alexa 647-labelled HBHA for 30 min on ice and the median fluorescence intensity of HBHA-bound cells was determined by flow cytometry. Data are representative of two independent experiments with two technical replicates each.

C. ELISA to determine interaction between HBHA and Sdc1 and Sdc4 (black bars). ELISA was performed with BSA instead of HBHA as control (white bars). D. Scramble control and Sdc KD A549 cells were cultured with WT or HBHA mutant BCG (MOI of 20) for $3 \mathrm{~h}$. Cells were lysed and diluted samples were plated onto agar plates. Data are representative of two or three independent experiments. ${ }^{* *}, P<0.01$; ${ }^{* \star}, P<0.005$; n.s., not significant using the one-way ANOVA (A, B) or the Bonferroni's post-test (D). Abbreviations: BCG, bacille Calmette-Guérin; CFU, colony-forming unit; HBHA, heparin-binding hemagglutinin adhesion; MFI: median fluorescence intensity; KD, knockdown; OD, optical density; Sdc, syndecan; WT, wild-type.

BCG. Control, Sdc1 or Sdc4 KD A549 cells were treated with BCG, and chemokines and cytokines were analysed by ELISA (Fig. 6). Inflammatory cytokines, including IL-12, TNF- $\alpha$, IL-1 $\beta$ and IL-6, were not detected in BCG-treated epithelial cell cultures. In contrast, IL-8 and MCP-1 were produced by BCG-treated cell lines and BCG-treated Sdc4 KD cells secreted these chemokines at lower concentrations compared to the control and Sdc1 KD cells (Fig. 6 A). Next, we examined the cell death of BCG-treated epithelial cells. Cell death of BCG-treated cell lines was induced after $48 \mathrm{~h}$, but was decreased in Sdc4 KD cells as compared to control and Sdc1 KD cells (Fig. $6 \mathrm{~B}$ ). In agreement with these data, the induction of chemokines and cell death by HBHA mutant BCG was reduced compared to WT BCG (Fig. $6 \mathrm{C}$ and D). We conclude that attachment to and internalization of BCG via Sdc4 contribute to the induction of inflammation and host cell death. 
Fig. 5. Ameliorated Mtb burden in lungs of Sdc $1 / 4^{-1-}$ DKO mice during early stage of infection.

A. Kinetics of $M$ tb colonization in Sdc1/ $4^{---}$DKO and C57BL/6J WT mice. Mice were aerosol infected with a low dose of H37Rv (200 CFUs). Mice were sacrificed and bacterial counts (CFUs) in lungs determined at days 1,7 and 15 p.i. as indicated.

B. Bacterial burdens in spleen (left) and liver (right) of mice sacrificed at day 15 p.i. C. Bacterial burden in lungs of mice infected i.v. with $1 \times 10^{5} \mathrm{CFUs}$ of H37Rv and sacrificed at day 1 p.i. Data show one representative out of two or three independent experiments, with at least five mice per time point. ${ }^{*}, P<0.05$; **, $P<0.01$; n.s. not significant using the Mann-Whitney test. Abbreviations: CFU, colony-forming unit; Sdc1/4 ${ }^{-1-}$ DKO, syndecan double knockout; p.i., postinfection; WT, wild-type.
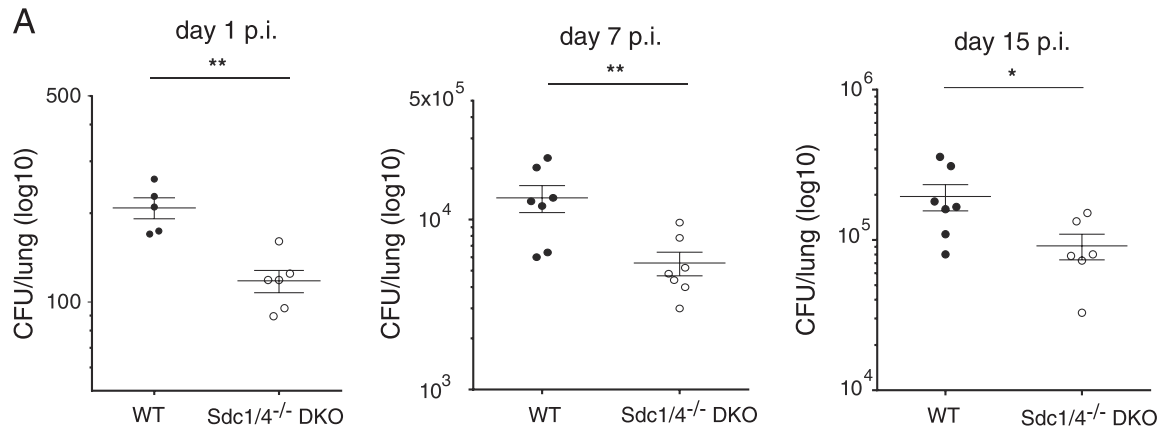

B
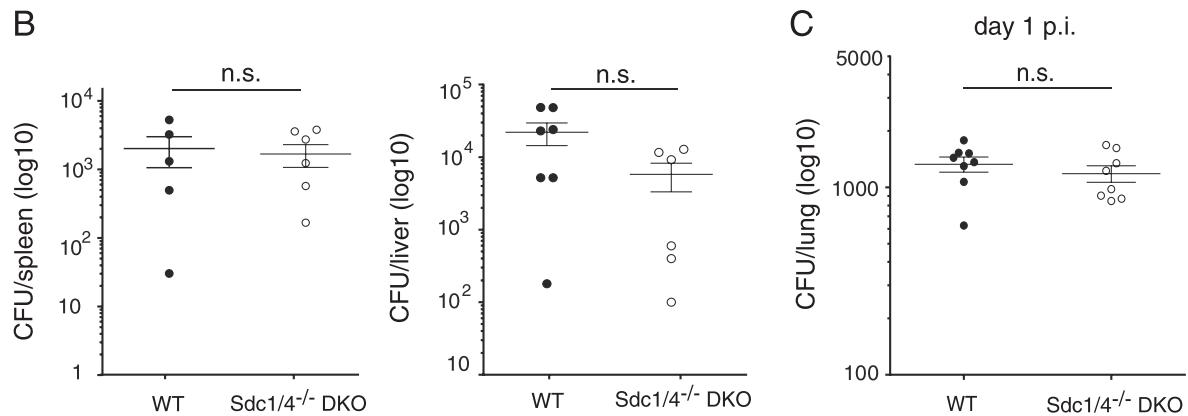

Fig. 6. Immune response of alveola epithelial cells.

$A$ and B. Scramble control and Sdc KD A549 cells were infected with BCG (MOI of 20) for $48 \mathrm{~h}$. Levels of the indicated chemokines and LDH activity in the culture supernatants were measured by ELISA and Cytotoxicity Detection Kit respectively.

C and D. A549 cells were cultured with WT or HBHA mutant BCG (MOI of 20 ) for $48 \mathrm{~h}$. Levels of the indicated chemokines and LDH activity in culture supernatants were measured by ELISA and Cytotoxicity Detection Kit

respectively. Data are representative of two independent experiments.

${ }^{\star}, P<0.05 ;{ }^{* *}, P<0.01 ;{ }^{* * *}, P<0.005$; n.s., not significant using the

Bonferroni's post-test. Abbreviations: BCG, bacille Calmette-Guérin; HBHA, heparin-binding hemagglutinin; KD, knockdown; Sdc, syndecan; WT, wildtype.
A
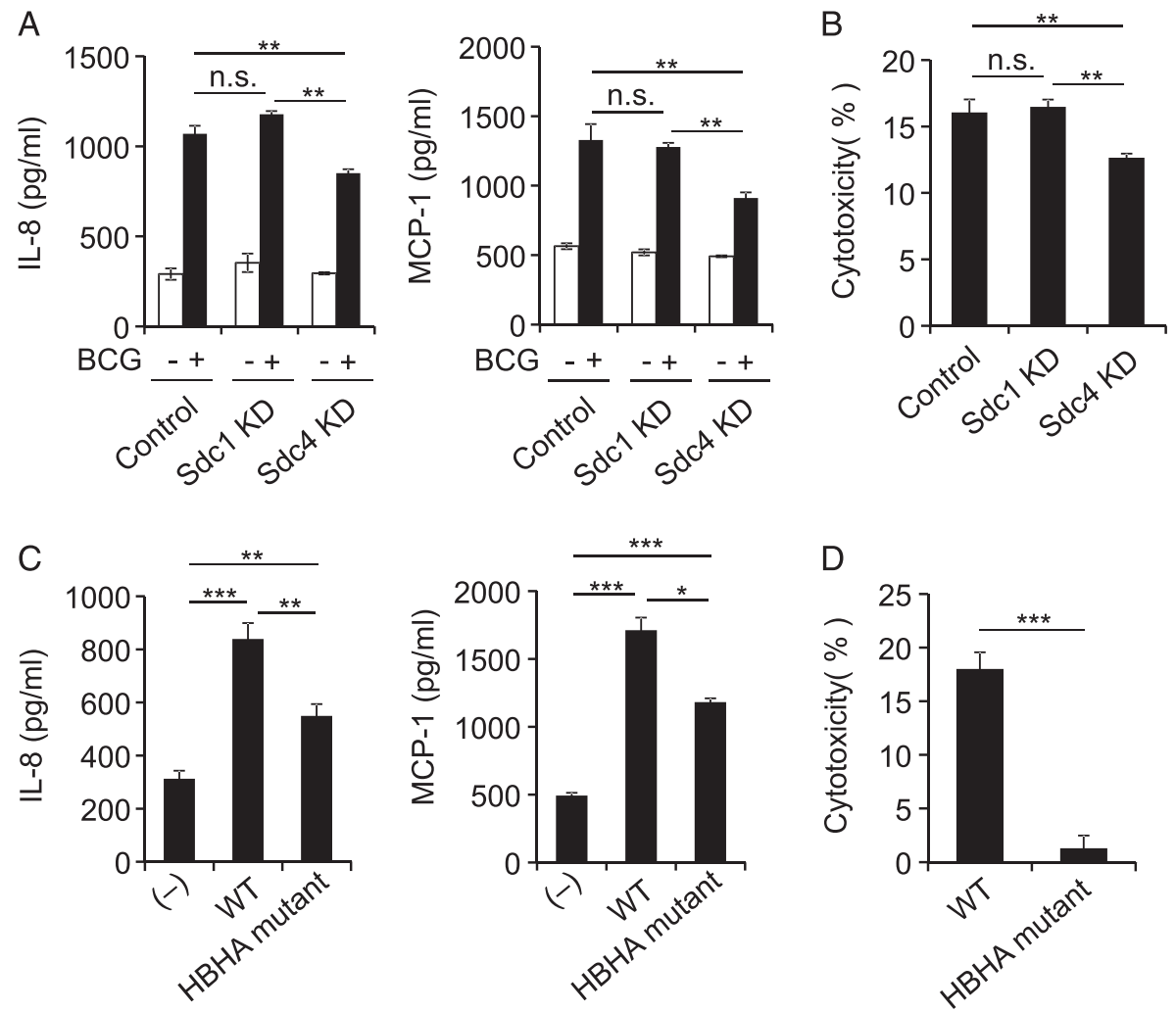

\section{Discussion}

Studies aimed at deciphering the early attachment and internalization mechanisms of $M t b$ in the lung have largely focused on the crosstalk between Mtb bacteria and macrophages and dendritic cells (Guirado et al., 2013; Lerner et al., 2015). Recent in vitro studies revealed that type II epithelial cells serve as reservoirs facilitating persistence of 
Mtb (Krishnan et al., 2010; Lerner et al., 2015). Accordingly, breaching of the alveolar epithelium could result in mycobacterial dissemination from the lung to distant organs (Krishnan et al., 2010; Russell, 2001). Few data, however, are available describing mechanisms underlying Mtb attachment to and internalization by epithelial cells (Krishnan et al., 2010). Moreover, the in vivo impact of the epithelial cell as a niche for Mtb persistence, and a source of spreading remains incompletely understood.

In this study we have identified a novel molecular mechanism for Mtb attachment to and internalization by lung epithelial cells. Distinct members of the proteoglycan family - the Sdcs - were identified as mediators of attachment to and internalization by airway epithelial cells for both Mtb and BCG. In a TB mouse model, Sdcs impacted on the course of TB at early time points p.i. with Mtb. Sdc1/Sdc4 ${ }^{-1-}$ DKO mice showed reduced lung colonization after aerosol exposure to $M t b$. Intriguingly, reduced $M t b$ load in the lungs of Sdc1/Sdc4 ${ }^{-1-}$ DKO mice was most pronounced at very early time points p.i., most notably as early as day 1 p.i. Moreover, after systemic infection, mycobacterial load in the lung of Sdc1/Sdc4 ${ }^{-1-}$ DKO mice was comparable to that of WT mice. Because the ameliorating effect of Sdc1/4 deletion was not because of differences in lung-resident innate cells and/or the recruitment of innate cells, our data provide compelling evidence for mycobacterial attachment to and internalization by the epithelial cell lining of the respiratory tract expressing Sdcs. Unique Sdcs seem to mediate adhesion of Mtb to the lining epithelium and facilitate its uptake by phagocytic cells. However, an additional intrinsic regulation in alveolar macrophages cannot be excluded, albeit our in vitro data do not support an essential role for Sdcs in mycobacterial internalization by macrophages.

Mycobacterial attachment and internalization upregulated the mRNA expression of Sdc4 in alveolar epithelial cells in a dose-dependent manner, whereas the mRNA expression of the other Sdc family members (Sdc1-Sdc3) remained largely unaltered. Therefore, Sdc4 seems to be the main mediator of mycobacterial attachment to and internalization by lung epithelial cells. Selective and specific targeting of a single member of the Sdc family was also shown in other types of infection. Whereas HEV clearly targets Sdc1, KD of Sdc4 did not inhibit viral binding to host cells (Kalia et al., 2009). Contrary to this, Sdc4 but not Sdc1 mediates HCV internalization as has been revealed by genetic KD experiments (Lefevre et al., 2014). Concurrent with the exclusive Sdc4 induction, the KD of Sdc4 in epithelial cells resulted in reduced mycobacterial attachment and internalization. Moreover, at later time points, Sdc4 mediated cell death of alveolar epithelial with potential implications for inflammation and tissue damage. Although our data suggest Sdc4 as important mediator of Mtb internalization by lung epithelial cells, single gene deletions of Sdc1 and Sdc4 in infected mice did not alter bacterial colonization of the lung, spleen or liver. Sdc1 and Sdc4 are expressed in steady state in the bronchoalveolar space and likely have redundant functions in tissues. This could explain the necessity of both molecules for restriction of Mtb in the lung. Importantly, Sdcs bind and mobilize surface and matrix proteins under homeostatic conditions and can thus modify the composition of the alveolar lining fluid (Pruessmeyer et al., 2010; Tan et al., 2012). Components of the alveolar fluid can largely influence clearance of the bacilli by neutrophils, which are recruited early during infection (Arcos et al., 2015). Furthermore, it is known that under homeostatic conditions, Sdc1 and Sdc4 are constitutively released by lung epithelial and smooth muscle cells and inflammation strongly increases this shedding into the bronchoalveolar fluid (Pruessmeyer et al., 2010; Tan et al., 2012). The presence of Sdc4 in lung fluids could have positive as well as negative implications in TB: binding of shed Sdc4 to mycobacteria could inhibit their binding to cell-surface Sdc4 and prevent adherence to epithelial cells, but it could also facilitate mycobacterial attachment to the mucus lining of the airways. Future experiments are needed to verify these notions.

$\mathrm{HBHA}$ is well appreciated as mycobacterial adhesin binding to heparan sulfate-containing proteoglycans (Menozzi et al., 1998; Mueller-Ortiz et al., 2002; Pethe et al., 2002). In this line, we identified HBHA as interaction partner for both Sdc4 and Sdc1. Binding of HBHA to epithelial cells was reduced when Sdc4 was deleted. Yet, our data suggest that $\mathrm{HBHA}$ is not the only mycobacterial adhesin binding to Sdcs on epithelial cells. First, the interaction between HBHA and Sdc4 or Sdc1 was of low affinity. Second, and more importantly, the role of HBHA and Sdc4 seems to be complementary, because in the absence of both molecules the infection of epithelial cells was further impaired when compared to the absence of either partner. This could explain the apparent discrepancy of our findings indicating a role for Sdcs in the initial pulmonary infection of Mtb in mice and the findings by Pethe et al. that deletion of $\mathrm{HBHA}$ in mycobacteria affects extrapulmonary dissemination but not colonization of the lung (Pethe et al., 2001).

$\mathrm{HBHA}$ interactions with glycosaminoglycan (GAG) sites have previously been postulated (Menozzi et al., 1996; Menozzi et al., 1998; Pethe et al., 2000) and proteoglycans other than Sdcs (i.e. glypicans) contain GAG sites. Additionally, other mycobacterial adhesins, such as the lamininbinding adhesin bind to extracellular proteoglycans (Kinhikar et al., 2006). Hence, we consider it most likely that the HBHA-Sdc interaction is only one way by which Mtb attachment to and internalization by lung epithelial cells is promoted. How distinctive the role for Sdcs is in comparison to other receptors such as glypicans or the extracellular matrix needs to be addressed in further studies. This apparent redundancy of molecular interaction between Mtb and 
epithelial cells can be interpreted to suggest that epithelial cells serve as important host niche for Mtb.

In conclusion, we here describe the targeting of Sdc family cognates as a strategy of Mtb for attachment to and internalization by lung epithelial cells. These molecules promote colonization and stable infection of the lung facilitating attachment to, and internalization by, epithelial cells. Hence, not only alveolar macrophages, but also respiratory epithelial cells are targeted by $M t b$ at early stages of infection. This novel entry mechanism could be exploited for future intervention strategies aimed at prevention of stable infection directly at the port of entry.

\section{Experimental procedures}

\section{Ethics statement}

The use of human biopsies was based on informed patient consent and approved by the Ethics Commission of Charite Hospital Berlin (Ulrichs et al., 2005). Sdc1 and Sdc4 immunohistochemistry staining was performed on healthy human trachea (Abcam) as described in the Supplementary Methods. All animal experiments were performed in accordance with the German Animal Protection Law (G 0063/11; T0087/13).

\section{Cells and bacteria}

M. bovis BCG (Denmark background), Ds-Red BCG (Saiga et al., 2015), WT M. bovis BCG, HBHA-mutant M. bovis BCG (Pasteur, 1173P2) and Mtb strain H37Rv (ATCC, no. 27294) were grown in Middlebrook $7 \mathrm{H} 9-\mathrm{ADC}$ medium and stored at $-80^{\circ} \mathrm{C}$ until use. Human alveolar epithelial A549 cells, human THP-1 cells, mouse type-II alveolar epithelial T7 cells and mouse BMDMs were cultured and differentiated as described in the Supplementary Methods.

\section{Quantitative real-time PCR}

Cells were infected with BCG [multiplicity of infection (MOI) of 1, 10, 50 and 100] for $3 \mathrm{~h}$, and then vigorously washed with phosphatebuffered saline (PBS) to eliminate extracellular BCG. After $24 \mathrm{~h}$, total RNA was isolated with Trizol (Invitrogen) and reverse-transcribed using the iScript cDNA Synthesis kit (Bio Rad). Quantitative realtime PCR was performed with the $\mathrm{ABI} 7900 \mathrm{H}$ system (Applied Biosystems) and using Sdc sequence primers (Table S1).

\section{Histology and immunohistochemistry}

Animals were aerosol-infected with $M$ tb, using a Glas-Col inhalation exposure system. Mouse lung was fixed with $4 \%$ paraformaldehyde (PFA) and embedded in paraffin. Formalin-fixed, paraffin-embedded lung tissues from patients with pulmonary TB were provided by the Central Tuberculosis Research Institute in Moscow through Dr. George Kosmiadi.

\section{Generation of Sdc KD cell lines}

Lentiviruses were produced according to TRC lentiviral proceedings (https://www.broadinstitute.org/genome_bio/trc/publicProtocols. html). HEK 293T cells were transfected with lentiviral packaging mix
(Sigma) and $100 \mathrm{ng}$ of shRNA (Table S2) containing pLKO.1-puro vector (Sigma) using Fugene 6 (Roche). For lentiviral infections, A549 cells were seeded one day prior to infection. Next day, cells were infected with $10 \mu \mathrm{l}$ of lentivirus in medium containing $8 \mu \mathrm{g} / \mathrm{ml}$ of polybrene (Sigma). Transduced cells were further selected using $5 \mu \mathrm{g} / \mathrm{ml}$ puromycin (Calbiochem). To determine the KD efficiency, total RNA was isolated with Trizol reagent and reverse-transcribed using the iScript cDNA Synthesis kit. Quantitative real-time PCR was performed using SYBR Green sequence primers (Table S1).

\section{Confocal microscopy}

WT $M$. bovis BCG and the HBHA-mutant $M$. bovis BCG strain were stained with $1 \mu \mathrm{M}$ Dil-C18 (Invitrogen) by incubation for $2 \mathrm{~h}$ prior to use. A549 cells were infected with Ds-Red BCG or Dil-C18 stained-BCG (MOI of 20 ) for $3 \mathrm{~h}$, vigorously washed with PBS and fixed with $4 \%$ PFA at $37^{\circ} \mathrm{C}$ for $15 \mathrm{~min}$. Cells were incubated with Alexa 488 phalloidin (Life Technologies) and DRAQ5 (Biostatus) for 20 min. Stained cells were mounted with ProLong Gold antifade reagent (Life Technologies) on glass slides and analysed using a fluorescence microscope (Leica TCS SP8). Fluorescence intensities were determined using ImageJ software.

\section{In vitro infection assay}

A549 cells were infected with BCG (MOI of 20) for $3 \mathrm{~h}$ or $\mathrm{Mtb} \mathrm{H} 37 \mathrm{Rv}$ (MOI of 1) for $30 \mathrm{~min}$ and $2 \mathrm{~h}$, and then vigorously washed with PBS. Cells were incubated with lysis buffer (0.5\% Triton X-100/PBS) for $5 \mathrm{~min}$. Harvested cells were serially diluted with dilution buffer (0.05\% Tween $80 / \mathrm{PBS})$ and plated on $7 \mathrm{H} 10$ or $7 \mathrm{H} 11-\mathrm{OADC}$ agar plates.

\section{Expression and purification of mycobacterial $\mathrm{HBHA}$}

Native HBHA expressed by M. bovis was kindly provided by Dr. Camille Locht (Institut Pasteur, Lille, France) and generated as previously described (Masungi et al., 2002). The plasmid for the expression of recombinant HBHA was kindly provided by Dr. Giovanni Delogu (University of Sacred Heart, Milano, Italy). The histidinetagged HBHA was expressed in a recombinant $M$. smegmatis strain as previously described (Delogu et al., 2004).

\section{Binding of labelled HBHA to epithelial cells}

For labelling of recombinant HBHA with Alexa 647 dye (Life Technologies), the protein was extensively dialysed in PBS overnight before use. Labelling was performed according to manufacturer's instructions (Life Technologies).

WT and KD cells were harvested as described above, counted and diluted to $10^{7}$ cells $/ \mathrm{ml}$. Subsequently, the cells were incubated with $2.5 \mu \mathrm{g}$ of HBHA conjugated to Alexa 647 for $1 \mathrm{~h}$ on ice, washed twice with PBS and analysed by flow cytometry.

\section{Enzyme-linked immunosorbent assay and cytotoxicity assay}

A549 cells were infected with BCG or HBHA-mutant BCG (MOI of 20) for $3 \mathrm{~h}$, vigorously washed with PBS and then supernatants were harvested after $48 \mathrm{~h}$. Levels of chemokines in culture supernatants 
were measured by enzyme-linked immunosorbent assay (ELISA), following manufacturer's instructions (R\&D Systems). Lactate dehydrogenase (LDH) activity was detected by Cytotoxicity Detection Kit according to manufacturer's instructions (Roche). The ELISA for detecting the interaction between HBHA and Sdc1 and Sdc4 was performed as described in the Supplementary Methods.

\section{Mice}

C57BL/6 (WT) mice were purchased from the Jackson Laboratory. $S d c 1^{-1-}$ and $S d c 4^{-l-}$ mice were kindly provided by $\mathrm{Dr}$. Frank Echtermeyer and Dr. Martin Götte (Alexander et al., 2000; Echtermeyer et al., 2001) and backcrossed for more than 12 generations with C57BL/6 mice. Sdc1/4 $4^{-1-}$ DKO mice were generated by mating $S d c 1^{-1-}$ and $S d c 4^{-1-}$ mice. WT, $S d c 1^{-1-}$, $S d c 4^{-1-}$ and $S d c 1 / 4^{-1-}$ littermates were bred and kept under specific pathogen-free conditions and infected mice were kept in a biosafety level 3 facility.

Animals were aerosol-infected with Mtb H37Rv using a Glas-Col inhalation exposure system at a low dose of approximately 200 CFUs per mouse. For intravenous infections, mice received $5 \times 10^{5} \mathrm{CFUs}$ of H37Rv into the tail vein. At designated time points, mice were sacrificed and the left lung lobe was homogenized in PBS containing $0.05 \%$ Tween 80 . Serial dilutions of the homogenates were plated onto Middlebrook $7 \mathrm{H} 11$ agar supplemented with $10 \%$ OADC (BD Biosciences) and ampicillin $(25 \mu \mathrm{g} / \mathrm{ml})$.

\section{Flow cytometry}

Single-cell suspensions (SCSs) were prepared from the lungs of naïve WT and Sdc1/4 ${ }^{-1-}$ DKO mice (Nouailles et al., 2014). Single cells were subsequently stained for surface markers by commercial anti-mouse antibodies according to manufacturer's instructions (see Supplementary Methods).

\section{Statistical analysis}

The statistical significance of data from in vitro experiments was evaluated using either unpaired $t$-test, or one-way ANOVA and Bonferroni's post-test for multiple measurements. CFU counts in lungs of mice were compared using the Mann-Whitney test. Values of $P<0.05$ were considered statistically significant.

\section{Acknowledgements}

We thank Frank Echtermeyer (Medizinische Hochschule Hannover, Germany) for providing Sdc4 ${ }^{-/-}$mice and Martin Götte (University of Muenster, Germany) for sharing Sdc $1^{-1-}$ mice. Moreover, we thank Giovanni Delogu (Catholic University of Sacred Heart, Milano, Italy) and Dr. Camille Locht (Institut Pasteur, Lille, France) for kindly providing the recombinant and native HBHA protein respectively. We also thank George Kosmiadi for help in providing clinical samples for this study. Finally, we thank Mary Louise Grossman for excellent help in preparing the manuscript.

\section{Funding}

HS was supported by The Uehara Memorial Foundation and Astellas Foundation for Research on Metabolic Disorders in
Japan. NZ was supported by the IMPRS Graduate School of the Max Planck Institute for Infection Biology in Berlin. This work received financial support from the European Union's Seventh Framework Programme (EU FP7) project 'ADITEC' (HEALTH-F4-2011-280873); the EU Horizon 2020 Research and Innovation Programme 'TBVAC 2020' (Grant no. 643381) and the German Federal Ministry of Education and Research (Bundesministerium fur Bildung und Forschung, BMBF) 'inVAC' (Grant no. 03ZZ0806A) to SHEK.

\section{Potential conflicts of interest}

All authors: No reported conflicts.

\section{References}

Alexander, C.M., Reichsman, F., Hinkes, M.T., Lincecum, J., Becker, K.A., Cumberledge, S., and Bernfield, M. (2000) Syndecan-1 is required for Wnt-1-induced mammary tumorigenesis in mice. Nat Genet 25: 329-332.

Andersen, P., and Kaufmann, S.H. (2014) Novel vaccination strategies against tuberculosis. Cold Spring Harb Perspect Med 4 4:a018523.

Arcos, J., Diangelo, L.E., Scordo, J.M., Sasindran, S.J., Moliva, J.I., Turner, J., and Torrelles, J.B. (2015) Lung mucosa lining fluid modification of Mycobacterium tuberculosis to reprogram human neutrophil killing mechanisms. J Infect Dis 212: 948-958.

Bacsa, S., Karasneh, G., Dosa, S., Liu, J., Valyi-Nagy, T., and Shukla, D. (2011) Syndecan-1 and syndecan-2 play key roles in herpes simplex virus type-1 infection. J Gen Virol 92: 733-743.

Bartlett, A.H., and Park, P.W. (2010) Proteoglycans in hostpathogen interactions: molecular mechanisms and therapeutic implications. Expert Rev Mol Med 12: e5.

Bermudez, L.E., and Goodman, J. (1996) Mycobacterium tuberculosis invades and replicates within type II alveolar cells. Infect Immun 64: 1400-1406.

Bermudez, L.E., and Sangari, F.J. (2001) Cellular and molecular mechanisms of internalization of mycobacteria by host cells. Microbes Infect 3: 37-42.

Bishop, J.R., Schuksz, M., and Esko, J.D. (2007) Heparan sulphate proteoglycans fine-tune mammalian physiology. Nature 446: 1030-1037.

Bobardt, M.D., Saphire, A.C., Hung, H.C., Yu, X., Van der Schueren, B., Zhang, Z., et al. (2003) Syndecan captures, protects, and transmits HIV to T lymphocytes. Immunity 18: 27-39.

Couchman, J.R. (2010) Transmembrane signaling proteoglycans. Annu Rev Cell Dev Biol 26: 89-114.

Delogu, G., and Brennan, M.J. (1999) Functional domains present in the mycobacterial hemagglutinin, HBHA. J Bacteriol 181: 7464-7469.

Delogu, G., Bua, A., Pusceddu, C., Parra, M., Fadda, G., Brennan, M.J., and Zanetti, S. (2004) Expression and purification of recombinant methylated HBHA in Mycobacterium smegmatis. FEMS Microbiol Lett 239: 33-39.

Dupres, V., Verbelen, C., Raze, D., Lafont, F., and Dufrene, Y.F. (2009) Force spectroscopy of the interaction between 
mycobacterial adhesins and heparan sulphate proteoglycan receptors. Chemphyschem 10: 1672-1675.

Echtermeyer, F., Streit, M., Wilcox-Adelman, S., Saoncella, S., Denhez, F., Detmar, M., and Goetinck, P. (2001) Delayed wound repair and impaired angiogenesis in mice lacking syndecan-4. J Clin Invest 107: R9-R14.

Freissler, E., Meyer auf der, H.A., David, G., Meyer, T.F., and Dehio, C. (2000) Syndecan-1 and syndecan-4 can mediate the invasion of OpaHSPG-expressing Neisseria gonorrhoeae into epithelial cells. Cell Microbiol 2: 69-82.

Gotte, M. (2003) Syndecans in inflammation. FASEB J 17: 575-591.

Guirado, E., Schlesinger, L.S., and Kaplan, G. (2013) Macrophages in tuberculosis: friend or foe. Semin Immunopathol 35: 563-583.

Kalia, M., Chandra, V., Rahman, S.A., Sehgal, D., and Jameel, S. (2009) Heparan sulfate proteoglycans are required for cellular binding of the hepatitis E virus ORF2 capsid protein and for viral infection. J Virol 83: 12714-12724.

Kinhikar, A.G., Vargas, D., Li, H., Mahaffey, S.B., Hinds, L., Belisle, J.T., and Laal, S. (2006) Mycobacterium tuberculosis malate synthase is a laminin-binding adhesin. Mol Microbiol 60: 999-1013.

Krishnan, N., Robertson, B.D., and Thwaites, G. (2010) The mechanisms and consequences of the extra-pulmonary dissemination of Mycobacterium tuberculosis. Tuberculosis (Edinb) 90: 361-366.

Lefevre, M., Felmlee, D.J., Parnot, M., Baumert, T.F., and Schuster, C. (2014) Syndecan 4 is involved in mediating $\mathrm{HCV}$ entry through interaction with lipoviral particleassociated apolipoprotein E. PLoS One 9: e95550.

Lerner, T.R., Borel, S., and Gutierrez, M.G. (2015) The innate immune response in human tuberculosis. Cell Microbiol 17: 1277-1285.

Masungi, C., Temmerman, S., Van Vooren, J.P., Drowart, A., Pethe, K., Menozzi, F.D., et al. (2002) Differential T and B cell responses against Mycobacterium tuberculosis heparinbinding hemagglutinin adhesin in infected healthy individuals and patients with tuberculosis. J Infect Dis 185: 513-520.

Menozzi, F.D., Rouse, J.H., Alavi, M., Laude-Sharp, M., Muller, J., Bischoff, R., et al. (1996) Identification of a heparin-binding hemagglutinin present in mycobacteria. J Exp Med 184: 993-1001.

Menozzi, F.D., Bischoff, R., Fort, E., Brennan, M.J., and Locht, C. (1998) Molecular characterization of the mycobacterial heparin-binding hemagglutinin, a mycobacterial adhesin. Proc Natl Acad Sci U S A 95: 12625-12630.

Menozzi, F.D., Reddy, V.M., Cayet, D., Raze, D., Debrie, A.S., Dehouck, M.P., et al. (2006) Mycobacterium tuberculosis heparin-binding haemagglutinin adhesin ( $\mathrm{HBHA})$ triggers receptor-mediated transcytosis without altering the integrity of tight junctions. Microbes Infect 8: 1-9.

Mueller-Ortiz, S.L., Sepulveda, E., Olsen, M.R., Jagannath, C., Wanger, A.R., and Norris, S.J. (2002) Decreased infectivity despite unaltered $\mathrm{C} 3$ binding by a DeltahbhA mutant of Mycobacterium tuberculosis. Infect Immun 70: 6751-6760.

Nishimura, J., Saiga, H., Sato, S., Okuyama, M., Kayama, H., Kuwata, H., et al. (2008) Potent antimycobacterial activity of mouse secretory leukocyte protease inhibitor. J Immunol 180: 4032-4039.
Nouailles, G., Dorhoi, A., Koch, M., Zerrahn, J., Weiner, J., III, Fae, K.C., et al. (2014) CXCL5-secreting pulmonary epithelial cells drive destructive neutrophilic inflammation in tuberculosis. J Clin Invest 124: 1268-1282.

Parra, M., Pickett, T., Delogu, G., Dheenadhayalan, V., Debrie, A.S., Locht, C., and Brennan, M.J. (2004) The mycobacterial heparin-binding hemagglutinin is a protective antigen in the mouse aerosol challenge model of tuberculosis. Infect Immun 72: 6799-6805.

Pethe, K., Aumercier, M., Fort, E., Gatot, C., Locht, C., and Menozzi, F.D. (2000) Characterization of the heparinbinding site of the mycobacterial heparin-binding hemagglutinin adhesin. J Biol Chem 275: 14273-14280.

Pethe, K., Alonso, S., Biet, F., Delogu, G., Brennan, M.J., Locht, C., and Menozzi, F.D. (2001) The heparin-binding haemagglutinin of $M$. tuberculosis is required for extrapulmonary dissemination. Nature 412: 190-194.

Pethe, K., Bifani, P., Drobecq, H., Sergheraert, C., Debrie, A.S., Locht, C., and Menozzi, F.D. (2002) Mycobacterial heparinbinding hemagglutinin and laminin-binding protein share antigenic methyllysines that confer resistance to proteolysis. Proc Natl Acad Sci U S A 99: 10759-10764.

Pieters, J. (2008) Mycobacterium tuberculosis and the macrophage: maintaining a balance. Cell Host Microbe 3: 399-407.

Pruessmeyer, J., Martin, C., Hess, F.M., Schwarz, N., Schmidt, S., Kogel, T., et al. (2010) A disintegrin and metalloproteinase 17 (ADAM17) mediates inflammation-induced shedding of syndecan-1 and -4 by lung epithelial cells. J Biol Chem 285 : 555-564.

Rivas-Santiago, B., Hernandez-Pando, R., Carranza, C., Juarez, E., Contreras, J.L., Aguilar-Leon, D., et al. (2008) Expression of cathelicidin LL-37 during Mycobacterium tuberculosis infection in human alveolar macrophages, monocytes, neutrophils, and epithelial cells. Infect Immun 76: 935-941.

Russell, D.G. (2001) TB comes to a sticky beginning. Nat Med 7: 894-895.

Saiga, H., Nishimura, J., Kuwata, H., Okuyama, M., Matsumoto, S., Sato, S., et al. (2008) Lipocalin 2-dependent inhibition of mycobacterial growth in alveolar epithelium. $J$ Immunol 181: 8521-8527.

Saiga, H., Nieuwenhuizen, N., Gengenbacher, M., Koehler, A.B., Schuerer, S., Moura-Alves, P., et al. (2015) The recombinant BCG deltaureC::hly vaccine targets the AIM2 inflammasome to induce autophagy and inflammation. $J$ Infect Dis 211: 1831-1841.

Shafti-Keramat, S., Handisurya, A., Kriehuber, E., Meneguzzi, G., Slupetzky, K., and Kirnbauer, R. (2003) Different heparan sulfate proteoglycans serve as cellular receptors for human papillomaviruses. J Virol 77: 13125-13135.

Shi, Q., Jiang, J., and Luo, G. (2013) Syndecan-1 serves as the major receptor for attachment of hepatitis $C$ virus to the surfaces of hepatocytes. J Virol 87: 6866-6875.

Tan, X., Khalil, N., Tesarik, C., Vanapalli, K., Yaputra, V., Alkhouri, H., et al. (2012) Th1 cytokine-induced syndecan-4 shedding by airway smooth muscle cells is dependent on mitogen-activated protein kinases. Am J Physiol Lung Cell Mol Physiol 302: L700-L710.

Teitelbaum, R., Schubert, W., Gunther, L., Kress, Y., Macaluso, F., Pollard, J.W., et al. (1999) The M cell as a portal of entry to the lung for the bacterial pathogen Mycobacterium tuberculosis. Immunity 10: 641-650. 
Ulrichs, T., Kosmiadi, G.A., Jorg, S., Pradl, L., Titukhina, M., Mishenko, V., et al. (2005) Differential organization of the local immune response in patients with active cavitary tuberculosis or with nonprogressive tuberculoma. J Infect Dis 192: 89-97.

WHO. Global Tuberculosis Report 2015. (2015) Geneva, WHO Press. Ref Type: Report

de Witte, L., Bobardt, M., Chatterji, U., Degeest, G., David, G., Geijtenbeek, T.B., and Gallay, P. (2007) Syndecan-3 is a dendritic cell-specific attachment receptor for HIV-1. Proc Natl Acad Sci U S A 104: 19464-19469.

Woods, A., Oh, E.S., and Couchman, J.R. (1998) Syndecan proteoglycans and cell adhesion. Matrix Biol 17: 477-483.

\section{Supporting information}

Additional supporting information may be found in the online version of this article at the publisher's web-site:

Table S1. qPCR primer sequences

Table S2. shRNA sequences

Fig. S1. mRNA expression in Sdc2 and Sdc3 KD cell lines in response to BCG. (A) A549 cells, T7 cells and THP-1 macrophages were cultured with BCG (MOI of 1, 10, 50 and 100) for $24 \mathrm{~h}$, and quantitative real-time PCR was performed. Data are representative of three independent experiments. (B) Lentiviruses were produced according to TRC lentiviral proceedings for the generation of Sdc KD cell lines. Data are shown as relative mRNA levels normalized to the corresponding GAPDH level. Data are representative of two independent experiments. ${ }^{*}, P<0.05$. Abbreviations: WT, wild-type; KD, knockdown; BCG, bacille CalmetteGuérin; Sdc, syndecan.

Fig. S2. Induction of Sdc1 and Sdc4 mRNA expression by Mtb. Bone-marrow-derived macrophages (BMDMs) were cultured with Mtb H37Rv (MOI of 1) for $4 \mathrm{~h}$ (left) or $28 \mathrm{~h}$ (right) and quantitative real-time PCR was performed. Data are representative of two independent experiments. ${ }^{* *} ; P<0.01$; n.s., not significant. Abbreviations: Sdc, syndecan.

Fig. S3. Sdc expression on the trachea and lung of mice and TB patients. (A) Sdc4 expression on tracheal epithelial cells of mouse lung tissue (left panel) and human lung tissue (right panel). (B) Mice were infected with low-dose Mtb H37Rv. Naive lung (top) and lung at day 15 p.i (middle) and 56 p.i. (bottom) are shown. A total of 2-3 specimens were analyzed for Sdc1 expression. (C) Lung biopsies from patients with active TB. Sdc1 staining of lung sections at different magnifications as indicated. A total of 6 specimens were analyzed.

Fig. S4. Involvement of Sdc4 in BCG attachment to versus internalization by lung epithelial cells. WT, Scramble control and Sdc KD A549 cells were infected with Ds-Red BCG [MOI of 20 (red)] for $3 \mathrm{~h}$. Extracellular bacteria were detected by a Cy5-conjugated anti-rabbit IgG secondary antibody (left). Cells were stained with phalloidin (green) and nucleus with DAPI (blue). Numbers of BCG staining positive for Cy5 or Ds-Red were quantified in an average of six random fields using ImageJ software (right). Representative data from two independent experiments. Abbreviations: KD; knockdown; Sdc, syndecan; WT, wild-type.

Fig. S5. Involvement of Sdc4 in Mtb attachment to and internalization by lung epithelial cells. (A) Scramble control and Sdc KD A549 cells were cultured with Mtb H37Rv (MOI of 1) for $30 \mathrm{~min}$ and $2 \mathrm{~h}$. Cells were lysed and samples were plated onto agar plates. Cumulative data from two independent experiments. (B) Scramble control and Sdc KD A549 cells were cultured with Ds-Red MTB [MOI of 20 (red)] for $3 \mathrm{~h}$. Cells were stained with phalloidin (green) and the nucleus with DAPI (blue). The percentage of cells harboring $M$ tb was quantified (bottom) by determining the fluorescence intensities of MTB bacteria in an average of six random fields using the ImageJ software. Data are representative of two independent experiments. (C) WT, Sdc $4^{-1-}$ and Sdc1 ${ }^{-1-}$ bone marrow-derived macrophages were cultured with $M$ tb $\mathrm{H} 37 \mathrm{Rv}$ [MOI of 5] for $4 \mathrm{~h}$. Cells were lysed and samples were plated onto agar plates. Data from one experiment with three technical replicates. ${ }^{*}, P<0.05$; ${ }^{* *} ; P<0.01$; ${ }^{* * *} ; P<0.005$; n.s., not significant. Abbreviations: MTB, Mycobacterium tuberculosis; CFUs, colony-forming units; KD; knockdown; Sdc, syndecan; WT, wild-type.

Fig. S6. Similar Mtb load in lungs of WT, Sdc1 $1^{-1-}$ and $\mathrm{Sdc} 4^{-1-}$ mice during early stages of infection. Sdc4 ${ }^{-1-}$ (A) or $\mathrm{Sdc} 1^{-1-} \mathrm{KO}$ mice $(\mathrm{B})$ were infected with a low aerosol dose of Mtb H37Rv (200 CFUs). At indicated time points p.i., mice were sacrificed and bacterial burden in the lungs determined. Data of one representative (day 15 p.i.) or two independent (day 1 p.i.) experiments. Abbreviations: p.i., post-infection; CFUs, colony forming units; WT, wild-type; Sdc, syndecan.

Fig. S7. Frequencies of innate cells in the lung of naive WT and Sdc1/4 ${ }^{-1-}$ DKO mice. Mice were sacrificed and lungs were harvested and homogenized to obtain singlecell suspensions. Innate cell populations in the lung were identified by flow cytometric analysis. (A) Gating strategy for all cell populations analyzed. (B) Comparison of percentages of PMNs, alveolar macrophages, lung macrophages, lung monocytes and DCs of total leukocytes in the lung of WT (black) and Sdc1/4 ${ }^{-1-}$ DKO mice (white). Representative data from two independent experiments with three mice each. Abbreviations: DCs, dendritic cells; PMNs, polymorphonuclear leukocytes; Sdc1/4 ${ }^{-/-}$DKO, syndecan double knockout; p.i., post-infection; WT, wild-type. 